\title{
Dirichlet forms and Markov semigroups on non-associative vector bundles
}

\section{Cho-Ho CHU and Zhongmin QIAN}

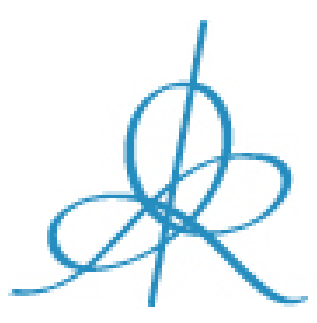

Institut des Hautes Études Scientifiques

35 , route de Chartres

91440 - Bures-sur-Yvette (France)

Février 2006

IHES/M/06/04 


\title{
DIRICHLET FORMS AND MARKOV SEMIGROUPS ON NON-ASSOCIATIVE VECTOR BUNDLES
}

\author{
CHO-HO CHU AND ZHONGMIN QIAN
}

\begin{abstract}
We introduce non-associative vector bundles and study Dirichlet forms and the associated Markov semigroups on these bundles.
\end{abstract}

\section{INTRODUCTION}

A non-commutative theory of Dirichlet forms and Markov semigroups has been developed in $[1,8,9,10]$. Two forms of non-commutative theory are usually considered: either the domains of the Dirichlet forms are furnished by some non-commutative $\mathrm{C}^{*}$-algebras, typically, the non-commutative $L^{p}(\mathcal{A})$ spaces of a semifinite von Neumann algebra $\mathcal{A}$, or, one considers the semigroups acting on sections of vector bundles over Riemannian manifolds, with non-commutative fibres. In $[9,10]$, the latter case has been studied for $\mathrm{C}^{*}$-bundles over compact manifolds whose fibres are finite-dimensional real $\mathrm{C}^{*}$-algebras. To be precise, the Dirichlet forms in both cases are defined in terms of the Hermitian part of the relevant spaces, namely, either the Hermitian part

$$
L_{h}^{2}(\mathcal{A})=\left\{x \in L^{2}(\mathcal{A}): x^{*}=x\right\}
$$

of the non-commutative space $L^{2}(\mathcal{A})$, as in [1, p. 177], or the section $L^{2}\left(\mathfrak{A}_{h}\right)$ with bundle $\mathfrak{A}_{h}$ whose fibres are the Hermitian part

$$
A_{h}=\left\{x \in A: x^{*}=x\right\}
$$

of a finite-dimensional real $\mathrm{C}^{*}$-algebra $A$, equipped with the $L_{2}$-norm of a trace, as in [9, Theorem 2]. It was also noted in [9] that a natural alternative approach would be to consider bundles whose fibres have the structure of a compact Jordan algebra.

In this paper, we consider more general vector bundles modelled on the nonassociative $L^{p}$-spaces, usually infinite dimensional, of a semifinite Jordan von Neumann algebra. This includes the bundles $\mathfrak{A}_{h}$ considered in [9] as well as the alternative approach proposed in [9] and mentioned above. We describe a framework for a non-associative theory of Dirichlet forms on these bundles and extend to this setting some contractivity results concerning the associated Markov semigroups (cf. $[9,10,17])$.

Mathematics Subject Classification 2000. 47D03, 58G32, 46L50, 46L70, 35J05, 31C05. 
We begin by describing the non-associative $L^{p}$-spaces, constructed from a Jordan algebra. We recall that a real, but not necessarily associative, algebra $\mathcal{A}$ is called a Jordan algebra if its algebraic product satisfies

$$
x y=y x \quad \text { and } \quad x^{2}(y x)=\left(x^{2} y\right) x \quad(x, y \in \mathcal{A}) .
$$

By a Jordan von Neumann algebra $\mathcal{A}$, we mean a real Banach space $\mathcal{A}$ which is also a Jordan algebra, with a (necessarily unique) separable predual $\mathcal{A}_{*}$, such that

$$
\begin{aligned}
\|x y\| & \leq\|x\|\|y\| \\
\left\|x^{2}\right\| & =\|x\|^{2} \\
\left\|x^{2}\right\| & \leq\left\|x^{2}+y^{2}\right\|
\end{aligned}
$$

for $x, y \in \mathcal{A}$. Without the separability condition on the predual, these algebras are known as $J B W$-algebras in literature [19]. The weak topology on $\mathcal{A}$ is the topology $\sigma\left(\mathcal{A}, \mathcal{A}_{*}\right)$. We note that $\mathcal{A}$ contains an identity $\mathbf{1}$ and the order in $\mathcal{A}$ is induced by the closed cone

$$
\mathcal{A}^{+}=\left\{x^{2}: x \in \mathcal{A}\right\}
$$

and we have $\mathcal{A}=\mathcal{A}^{+}-\mathcal{A}^{+}$. Given $x \in \mathcal{A}$, one can define its modulus $|x|=$ $\left(x^{2}\right)^{1 / 2} \in \mathcal{A}^{+}$. Each $x \in \mathcal{A}$ has a polar decomposition

$$
x=s|x|
$$

where $s$ is a symmetry in $\mathcal{A}$ which means that $s^{2}=\mathbf{1}$.

Example 1.1. Let $\mathcal{A}$ be a (complex) von Neumann algebra with a separable predual, for instance, the algebra $B(H)$ of bounded linear operators on a complex separable Hilbert space $H$. Then the Hermitian part

$$
\mathcal{A}_{h}=\left\{T \in \mathcal{A}: T^{*}=T\right\}
$$

is a Jordan von Neumann algebra, with the Jordan product defined by

$$
T \circ S=\frac{1}{2}(T S+S T)
$$

where the product on the right is the original product in $\mathcal{A}$. The positive cone $\mathcal{A}^{+}=\left\{T^{*} T: T \in \mathcal{A}\right\}$ coincides with $\mathcal{A}_{h}^{+}$.

Example 1.2. Let $A$ be a real $C^{*}$-algebra. Then its complexification $\widetilde{A}=A+i A$ can be given a norm so that it becomes a (complex) $\mathrm{C}^{*}$-algebra, and $A$ embeds isometrically as a real $C^{*}$-subalgebra of $\widetilde{A}[15,15.4]$. We note that $A$ is generally not identical with the Hermitian part of $\widetilde{A}$. If $A$ has a separable predual, then its Hermitian part

$$
A_{h}=\left\{x \in A: x^{*}=x\right\}
$$

is a Jordan von Neumann algebra, with the Jordan product defined by

$$
x \circ y=\frac{1}{2}(x y+y x)
$$

where the associative product on the right is the original product in $A$. 
We refer to [19] for other examples of Jordan von Neumann algebras which are not the Hermitian part of a real or complex $\mathrm{C}^{*}$-algebra.

We recall that a Jordan von Neumann algebra $\mathcal{A}$ is semifinite if it admits a faithful semifinite normal trace. A trace on $\mathcal{A}$ is an additive function $\tau: \mathcal{A}^{+} \longrightarrow$ $[0, \infty]$ satisfying

(i) $\tau(\alpha x)=\alpha \tau(x) \quad(\alpha \geq 0)$

(ii) $\tau(s x s)=\tau(x) \quad$ ( $s$ is a symmetry).

A trace $\tau$ is faithful if $\tau(x)=0$ implies $x=0$. It is called semifinite if for any $x \in \mathcal{A}^{+} \backslash\{0\}$, there exists $y \in \mathcal{A}^{+} \backslash\{0\}$ such that $y \leq x$ and $\tau(y)<\infty$. If $\tau$ preserves monotone convergence, then it is called normal.

A prototypic example of a semifinite Jordan von Neumann algebra is the Hermitian part $B(H)_{h}$ of the algebra $B(H)$ of bounded operators on a separable Hilbert space $H$, with the canonical trace; but important examples include Hermitian parts of all finite von Neumann algebras with separable predual, in particular, the group von Neumann algebras of infinite-conjugacy-class groups which are type $\mathrm{II}_{1}$ factors (cf. [27, p.367]).

In the sequel, $\mathcal{A}$ will denote a semifinite Jordan von Neumann algebra with a faithful semifinite normal trace $\tau$. There is a weakly dense ideal of $\mathcal{A}$ associated with $\tau$, namely,

$$
\mathcal{N}_{\tau}=\mathcal{N}_{\tau}^{+}-\mathcal{N}_{\tau}^{+}
$$

where

$$
\mathcal{N}_{\tau}^{+}=\left\{a \in \mathcal{A}^{+}: \tau(a)<\infty\right\}
$$

and the trace $\tau$ can be extended to a linear functional on $\mathcal{N}_{\tau}$, still denoted by $\tau$. For $1 \leq p<\infty$, we define the $L^{p}$-norm

$$
|||x| \|_{p}=\tau\left(|x|^{p}\right)^{1 / p} \quad\left(x \in \mathcal{N}_{\tau}\right)
$$

where $|x|^{p} \in \mathcal{N}_{\tau}^{+}$is defined by function calculus. The completion of the normed space $\left(\mathcal{N}_{\tau},|||\cdot| \|_{p}\right)$ is denoted by $L^{p}(\mathcal{A}, \tau)$, called the non-associative $L^{p}$-space of $\mathcal{A}$ with respect to $\tau$. The space $L^{1}(\mathcal{A}, \tau)$ is linearly isometric to $\mathcal{A}_{*}$ and $L^{2}(\mathcal{A}, \tau)$ is a Hilbert space with inner product denoted by $\langle\cdot, \cdot\rangle_{\tau}$. We define $L^{\infty}(\mathcal{A}, \tau)=\mathcal{A}$ and refer to [20] for further details of these $L^{p}$ spaces.

One can construct a non-commutative $L^{p}$-space $L^{p}\left(\mathcal{M}, \tau_{0}\right)$ of a (complex) von Neumann algebra $\mathcal{M}$ with a faithful semifinite normal trace $\tau_{0}$. If $\mathcal{M}$ has a separable predual, then the Hermitian part $\mathcal{A}=\mathcal{M}_{h}$ of $\mathcal{M}$ is a Jordan von Neumann algebra with trace $\tau$ which is the restriction of $\tau_{0}$ to $\mathcal{A}^{+}$, and $L^{p}(\mathcal{A}, \tau)$ identifies with the Hermitian part $L_{h}^{p}\left(\mathcal{M}, \tau_{0}\right)$ of $L^{p}\left(\mathcal{M}, \tau_{0}\right)[2]$.

Example 1.3. If $\mathcal{A}=\mathcal{B}(H)_{h}$ is the Hermitian part of the algebra of bounded operators on a separable Hilbert space $H$, with the canonical trace $\tau$, then $L^{2}(\mathcal{A}, \tau)=$ $\mathcal{N}_{\tau}$ is the space of self-adjoint Hilbert-Schmidt operators on $H$ and is separable.

Example 1.4. If $A$ is a finite-dimensional real $\mathrm{C}^{*}$-algebra, then $L^{2}\left(A_{h}, \tau\right)=$ $\left(A_{h},\left|\|\cdot \mid\|_{2}\right)\right.$ for any trace $\tau$ on $A_{h}$. This is the space considered in [9]. 


\section{Non-Associative Vector BUNDLES AND DiRIChlet FORMS}

In this section, we introduce non-associative vector bundles on Riemannian manifolds and the setting for a non-associative theory of Dirichlet forms. These bundles are vector bundles whose fibres have Jordan algebraic structures, more precisely, the fibres of these bundles are real Hilbert spaces isometric to a nonassociative Hilbert space of a semifinite Jordan von Neumann algebra.

Throughout, let $M$ be a Riemannian manifold equipped with a $\sigma$-finite Borel measure $\mu$. Let $L^{2}(\mathcal{A}, \tau)$ be a non-associative Hilbert space as before. We denote by $L^{2}\left(M, L^{2}(\mathcal{A}, \tau)\right)$ the real Hilbert space of (equivalence classes of) $L^{2}(\mathcal{A}, \tau)$ valued Bochner integrable functions $f$ on $M$ satisfying

$$
\|f\|_{2}=\left(\int_{M}\left|\|f(x) \mid\|_{2}^{2} d \mu(x)\right)^{\frac{1}{2}}<\infty\right.
$$

(cf. [13, p.97]), with inner product

$$
\langle f, g\rangle=\int_{M}\langle f(x), g(x)\rangle_{\tau} d \mu(x) .
$$

Let $C_{c}^{\infty}\left(M, L^{2}(\mathcal{A}, \tau)\right)$ be the space of smooth $L^{2}(\mathcal{A}, \tau)$-valued functions on $M$ with compact support. Standard arguments show that $C_{c}^{\infty}\left(M, L^{2}(\mathcal{A}, \tau)\right)$ is $\|\cdot\|_{2}$-dense in $L^{2}\left(M, L^{2}(\mathcal{A}, \tau)\right)$.

A vector bundle $\pi: E \longrightarrow M$ is called a non-associative bundle if its fibres $E_{x}$ are all real Hilbert spaces linearly isometric to the non-assoicative Hilbert space $L^{2}(\mathcal{A}, \tau)$ of a Jordan von Neumann algebra $\mathcal{A}$ with a faithful semifinite normal trace $\tau$. In this case, $E$ is a Hilbert manifold modeled on the real Hilbert space $L^{2}(\mathcal{A}, \tau) \times \mathbb{R}^{n}$ where $n=\operatorname{dim} M$. We denote the inner product in $E_{x}$ by $\langle\cdot, \cdot\rangle_{x}$. Given the linear isometry

$$
\gamma_{x}: E_{x} \longrightarrow L^{2}(\mathcal{A}, \tau)
$$

we have $\langle\xi, \zeta\rangle_{x}=\left\langle\gamma_{x}(\xi), \gamma_{x}(\zeta)\right\rangle_{\tau}$. The set $C_{c}^{\infty}(E)$ of smooth sections on $M$ with compact support is a vector space with inner product and norm:

$$
\begin{gathered}
\langle\varphi, \psi\rangle=\int_{M}\langle\varphi(x), \psi(x)\rangle_{x} d \mu(x) \\
\|\varphi\|_{2}=\langle\varphi, \varphi\rangle^{1 / 2} .
\end{gathered}
$$

The completion $\mathcal{L}^{2}(E)$ of $C_{c}^{\infty}(E)$ with respect to the above norm identifies with the real Hilbert space $L^{2}\left(M, L^{2}(\mathcal{A}, \tau)\right)$. More generally, for $1 \leq p<\infty$, we denote by $\mathcal{L}^{p}(E)$ the completion of $C_{c}^{\infty}(E)$ with respect to the following norm:

$$
\|\varphi\|_{p}=\left(\int_{M}\langle\varphi(x), \varphi(x)\rangle_{x}^{p / 2} d \mu(x)\right)^{1 / p} .
$$

Let $\mathcal{L}^{\infty}(E)$ be the space of (essentially) bounded sections on $M$.

The $L^{p}$-space $L^{p}(\mathcal{A}, \tau)$ can be partially ordered by the cone $L^{p}(\mathcal{A}, \tau)^{+}$which is defined to be the $\left|\|\cdot \mid\|_{p}\right.$-closure of $\mathcal{N}_{\tau}^{+}$. For $p \in(1, \infty)$, the norm $|\|\cdot \mid\|_{p}$ is Fréchet 
differentiable except at 0 . Given a map $f: \mathbb{R} \longrightarrow L^{p}(\mathcal{A}, \tau)^{+}$, differentiable at $t_{0} \in \mathbb{R}$ with $f\left(t_{0}\right) \neq 0$, we have, by [20, Lemma 14$]$,

$$
\left.\frac{d}{d t} \tau\left(f(t)^{p}\right)\right|_{t=t_{0}}=p \tau\left(\left.f\left(t_{0}\right)^{p-1} \frac{d}{d t} f(t)\right|_{t=t_{0}}\right) .
$$

For $z, w \in L^{2}(\mathcal{A}, \tau)^{+}$, we have $\langle z, w\rangle_{\tau} \geq 0$ (cf. [20, Lemma 1]). Every $z \in$ $L^{2}(\mathcal{A}, \tau)$ has a decomposition $z=z^{+}-z^{-}$with $z^{+}, z^{-} \geq 0$ and $z^{+} z^{-}=0$. The modulus of $z$ is defined to be $|z|=z^{+}+z^{-}$.

Each fibre $E_{x}$ of the non-associative vector bundle $\pi: E \longrightarrow M$ carries the above order and Jordan algebraic structures of $L^{2}(\mathcal{A}, \tau)$ via the isometry $\gamma_{x}$ : $E_{x} \longrightarrow L^{2}(\mathcal{A}, \tau)$. A section $\varphi$ of $E$ is said to be positive if $\varphi(x) \geq 0$ for almost all $x \in M$. We denote this by $\varphi \geq 0$.

Let $\Gamma(E)$ be the space of smooth sections of $E$. Given $\varphi \in \Gamma(E)$, we define $\varphi^{ \pm}(x)=\varphi(x)^{ \pm}$and $|\varphi|(x)=|\varphi(x)|$ for $x \in M$. Then $\varphi=\varphi^{+}-\varphi^{-}$and $|\varphi|=$ $\varphi^{+}+\varphi^{-}$. We have

$$
\left\langle\varphi^{+}, \varphi^{-}\right\rangle=\int_{M}\left\langle\varphi(x)^{+}, \varphi(x)^{-}\right\rangle_{x} d \mu(x)=0 .
$$

The above order structures can be extended to the completion $\mathcal{L}^{2}(E) \simeq L^{2}\left(M, L^{2}(\mathcal{A}, \tau)\right)$. A linear map $P: \mathcal{L}^{2}(E) \longrightarrow \mathcal{L}^{2}(E)$ is called positive, in symbol, $P \geq 0$, if $\varphi \geq 0$ implies $P \varphi \geq 0$.

Let $Q$ be a closable non-negative quadratic form with domain $C_{c}^{\infty}(E) \subset$ $\mathcal{L}^{2}(E)$. Then there is a positive self-adjoint operator $L$ in $\mathcal{L}^{2}(E)$ such that

$$
Q(\varphi, \psi)=\langle L \varphi, \psi\rangle \quad\left(\varphi, \psi \in C_{c}^{\infty}(E)\right)
$$

where we use the same symbol $Q$ for the associated symmetric bilinear form. We denote by $\mathcal{D}(L)$ the domain of $L$.

The proof of the following result is similar to [9, Theorem 1].

Theorem 2.1. Let $Q(\cdot)=\left\langle L^{1 / 2}(\cdot), L^{1 / 2}(\cdot)\right\rangle$ be a quadratic form where $L: \mathcal{D}(L) \longrightarrow$ $\mathcal{L}^{2}(E)$ is a self-adjoint, positive operator which generates a semigroup $\left(P_{t}\right)_{t \geq 0}$ on $\mathcal{L}^{2}(E)$. The following conditions are equivalent.

(i) $P_{t} \geq 0$ for $t>0$.

(ii) Given $\varphi \in \mathcal{D}\left(L^{1 / 2}\right)$, we have $|\varphi| \in \mathcal{D}\left(L^{1 / 2}\right)$ and $Q(|\varphi|) \leq Q(\varphi)$.

(iii) Given $\varphi \in \mathcal{D}\left(L^{1 / 2}\right)$, we have $|\varphi| \in \mathcal{D}\left(L^{1 / 2}\right)$ and $Q\left(\varphi^{+}, \varphi^{-}\right) \leq 0$.

(iv) For $\varphi \in \mathcal{L}^{2}(E)$ and $\varphi \geq 0$, we have $(\alpha+L)^{-1}(\varphi) \geq 0$ for all $\alpha>0$.

Proof. (i) $\Rightarrow$ (ii). Let $\varphi \in \mathcal{D}\left(L^{1 / 2}\right)$. Then by positivity of $P_{t}$, we have

$$
\begin{aligned}
\left\langle P_{t} \varphi, \varphi\right\rangle & =\left\langle P_{t} \varphi^{+}-P_{t} \varphi^{-}, \varphi^{+}-\varphi^{-}\right\rangle \\
& =\left\langle P_{t} \varphi^{+}, \varphi^{+}\right\rangle+\left\langle P_{t} \varphi^{-}, \varphi^{-}\right\rangle-\left\langle P_{t} \varphi^{+}, \varphi^{-}\right\rangle-\left\langle P_{t} \varphi^{-}, \varphi^{+}\right\rangle \\
& \leq\left\langle P_{t}|\varphi|,|\varphi|\right\rangle .
\end{aligned}
$$

Hence

$$
\frac{1}{t}\left\langle\left(I-P_{t}\right)|\varphi|,|\varphi|\right\rangle \leq \frac{1}{t}\left\langle\left(I-P_{t}\right) \varphi, \varphi\right\rangle
$$


and $\lim \sup _{t \rightarrow 0} \frac{1}{t}\left\langle\left(I-P_{t}\right)|\varphi|,|\varphi|\right\rangle \leq\left\langle L^{1 / 2} \varphi, L^{1 / 2} \varphi\right\rangle$. It follows that $|\varphi| \in \mathcal{D}\left(L^{1 / 2}\right)$ and $Q(|\varphi|) \leq Q(\varphi)$.

(ii) $\Leftrightarrow$ (iii). This follows from

$$
4 Q\left(\varphi^{+}, \varphi^{-}\right)=Q(|\varphi|)-Q(\varphi)
$$

where $\varphi,|\varphi| \in \mathcal{D}\left(L^{1 / 2}\right)$ implies that $\varphi^{ \pm} \in \mathcal{D}\left(L^{1 / 2}\right)$.

(iii) $\Rightarrow$ (iv). Fix $\alpha>0$. Denote $K=\mathcal{D}\left(L^{1 / 2}\right)$ which is a Hilbert space with respect to the inner product

$$
\langle\psi, \varphi\rangle_{1}=\left\langle L^{1 / 2} \psi, L^{1 / 2} \varphi\right\rangle+\alpha\langle\psi, \varphi\rangle .
$$

Let $J: K \longrightarrow \mathcal{L}^{2}(E)$ be the natural embedding. Then, for $\psi \in K, \varphi \in \mathcal{L}^{2}(E)$, we have

$$
\begin{aligned}
\left\langle\psi,(\alpha+L)^{-1} \varphi\right\rangle_{1}= & \left\langle L^{1 / 2} \psi, L^{1 / 2}(\alpha+L)^{-1} \varphi\right\rangle \\
& +\alpha\left\langle\psi,(\alpha+L)^{-1} \varphi\right\rangle \\
= & \left.\left\langle(\alpha+L) \psi,(\alpha+L)^{-1} \varphi\right)\right\rangle \\
= & \langle\psi, \varphi\rangle=\langle J \psi, \varphi\rangle .
\end{aligned}
$$

Therefore $J^{*} \varphi=(\alpha+L)^{-1} \varphi$. Let $\psi=J^{*} \varphi$. We have

$$
\begin{aligned}
\langle|\psi|,|\psi|\rangle_{1} & =Q(|\psi|)+\alpha\langle|\psi|,|\psi|\rangle \\
& \leq Q(\psi)+\alpha\langle\psi, \psi\rangle=\langle\psi, \psi\rangle_{1} .
\end{aligned}
$$

Let $\varphi \geq 0$. Then

$$
\begin{aligned}
\langle|\psi|, \psi\rangle_{1} & =\left\langle|\psi|, J^{*} \varphi\right\rangle_{1} \\
& =\langle|\psi|, \varphi\rangle \\
& \geq\langle\psi, \varphi\rangle=\left\langle\psi, J^{*} \varphi\right\rangle_{1}=\langle\psi, \psi\rangle_{1} .
\end{aligned}
$$

Hence $(\alpha+L)^{-1} \varphi=J^{*} \varphi=\psi=|\psi| \geq 0$.

(iv) $\Rightarrow$ (i). This follows from

$$
P_{t}=\lim _{n \rightarrow \infty}\left(I+\frac{t}{n} L\right)^{-n} .
$$

A quadratic form $Q$ in $\mathcal{L}^{2}(E)$ satisfying the conditions in Theorem 2.1 and generating a contractive semigroup $\left(P_{t}\right)$ on $\mathcal{L}^{p}(E)$ for $p \in[1, \infty]$ is called a Dirichlet form, where $P_{t}$ is called a contraction on $\mathcal{L}^{p}(E)$ if it maps $\mathcal{L}^{2}(E) \cap \mathcal{L}^{p}(E)$ into $\mathcal{L}^{2}(E) \cap \mathcal{L}^{p}(E)$, and is contractive in the $L^{p}$-norm.

From now on, we fix a non-associative vector bundle $\pi: E \longrightarrow M$ with fibres isometric to the real Hilbert space $L^{2}(\mathcal{A}, \tau)$ of a Jordan von Neumann algebra $\mathcal{A}$ with a faithful semifinite normal trace $\tau$. By [21, Theorem 1.8.19], the vector 
bundle $\pi: E \longrightarrow M$ has a Riemannian metric, that is, the inner product $\langle\cdot, \cdot\rangle_{x}$ on $E_{x}$ can be chosen to depend smoothly on $x \in M$. Let $T E$ be the total tangent space of $E$. By [21, Theorem 1.8.23], the above vector bundle possesses a metric connection $K: T E \longrightarrow E$, compatible with the Riemannian structure such that, for each $\varphi \in \Gamma(E)$,

$$
D_{X} \varphi(x):=K \circ d \varphi_{x}(X) \in E
$$

is the associated covariant derivation of $\varphi$ in the direction $X \in T_{x} M$, where $d \varphi_{x}$ : $T_{x} M \longrightarrow T_{\varphi(x)} E$ is the differential of $\varphi$ at $x \in M$. For any vector field $X$ on $M$, $D_{X} \varphi$ is a smooth section of $E$ (cf.[21, p.49]) and

$$
X\langle\varphi, \psi\rangle=\left\langle D_{X} \varphi, \psi\right\rangle+\left\langle\varphi, D_{X} \psi\right\rangle .
$$

We note that $K \circ d \varphi_{x} \in L\left(T_{x} M, E_{x}\right)$, the space of linear maps between $T_{x} M$ and $E_{x}$, and the tensor product $E_{x} \otimes T_{x}^{*} M$ is dense in $L\left(T_{x} M, E_{x}\right)$ in the compact open topology (cf. [13, p.240]). If the fibre $E_{x}$ is finite-dimensional, then $L\left(T_{x} M, E_{x}\right)=$ $E_{x} \otimes T_{x}^{*} M$ and we have the connection $D: \Gamma(E) \longrightarrow \Gamma(E) \otimes \Gamma\left(T^{*} M\right)$ given by

$$
D \varphi=K \circ d \varphi .
$$

For $\varphi, \psi \in C_{c}^{\infty}(E)$, we define

$$
\langle D \varphi(x), D \psi(x)\rangle_{\tau}=\sum_{i=1}^{n}\left\langle D_{X_{i}} \varphi(x), D_{X_{i}} \psi(x)\right\rangle_{x}
$$

where $\left\{X_{1}, \ldots, X_{n}\right\}$ is an orthonormal moving frame on $M$.

Given $\pi: E \longrightarrow M$ endowed with a Riemannian structure and a compatible connection $D$, the qudratic form

$$
\mathcal{E}(\varphi, \psi)=\int_{M}\langle D \varphi, D \psi\rangle_{\tau} d \mu \quad\left(\varphi, \psi \in C_{c}^{\infty}(E)\right)
$$

satisfies the conditions in Theorem 2.1 since $\mathcal{E}\left(\varphi^{+}, \varphi^{-}\right)=0$.

\section{HYPERCONTRACTIVITY}

The theory of hypercontractive semigroups was introduced in a fundamental paper of Nelson [24] who discovered that the Ornstein-Uhlenbeck semigroup $P_{t}$ : $L^{p}\left(\mathbb{R}^{d}, \mu\right) \longrightarrow L^{q}\left(\mathbb{R}^{d}, \mu\right)$ is bounded if $p, q$ and $t$ are properly related, where $\mu$ is the Gaussian measure. After important improvements in $[14,26]$, the precise minimum time $t$ for contractivity from $L^{p}$ to $L^{q}$ was established in [25].

In his seminal paper [17], Gross proved the equivalence of hypercontractivity and a logarithmic Sobolev inequality for diffusion semigroups which may be stated as follows. Let $\left(P_{t}\right)_{t \geq 0}$ be the diffusion semigroup associated to a local Dirichlet form $(\mathcal{E}, \mathcal{F})$ on $L^{2}(X, \mathcal{X}, \mu)$ for some $\sigma$-finite measure space $(X, \mathcal{X}, \mu)$. Let

$$
\operatorname{Ent}(f)=\int_{X}(f \ln f) d \mu-\left(\int_{X} f d \mu\right)\left(\ln \int_{X} f d \mu\right)
$$


denote the entropy of $f$. Let $a>0$ and $b \geq 0$. Define

$$
p(t)=1+(p-1) e^{4 t / a} ; \quad m(t)=b\left(p^{-1}-p(t)^{-1}\right) .
$$

Then the following logarithmic Sobolev inequality

$$
\operatorname{Ent}\left(f^{2}\right) \leq a \mathcal{E}(f, f)+b\|f\|_{2}^{2} \quad(f \in \mathcal{F})
$$

holds if, and only if,

$$
\left\|P_{t} f\right\|_{p(t)} \leq e^{m(t)}\|f\|_{p}
$$

for all $f \in L^{p}(X, \mathcal{X}, \mu), p \in(1, \infty)$ and $t>0$. We refer to $[3,6,11,12,17,18]$ for the evolution of this form of Gross's theorem. We also refer to [7] for a bibliographic review of hyercontractivity.

Let $\pi: E \rightarrow M$ be a non-associative vector bundle, endowed with a Riemannian structure and a compatible connection $D$. Let

$$
\mathcal{E}(\varphi, \psi)=\int_{M}\langle D \varphi, D \psi\rangle_{\tau} d \mu \quad\left(\varphi, \psi \in C_{c}^{\infty}(E) \subset \mathcal{L}^{2}(E)\right)
$$

be a Dirichlet form. Let $\left(P_{t}\right)_{t \geq 0}$ be the diffusion semigroup of the vector bundle $E$ with generator $L$ defined by $\overline{\mathcal{E}}$. That is, $P_{t}=e^{-t L}$ and the self-adjoint operator $L$ is determined via integration by parts

$$
\int_{M}\langle D \varphi, D \psi\rangle_{\tau} d \mu=\int_{M}\langle L \varphi, \psi\rangle_{\tau} d \mu .
$$

As $\mathcal{L}^{2}(E) \simeq L^{2}\left(M, L^{2}(A, \tau)\right)$, each $\varphi \in \mathcal{L}^{2}(E)$ identifies with a function in $L^{2}\left(M, L^{2}(A, \tau)\right)$ and we define

$$
|\varphi|_{\tau}(x)=\langle\varphi(x), \varphi(x)\rangle_{\tau}^{1 / 2} \quad(x \in M)
$$

which is abbreviated to $|\varphi|_{\tau}^{2}=\langle\varphi, \varphi\rangle_{\tau}$ if no confusion is likely. As before, let $\|\varphi\|_{p}$ denote the $L^{p}$-norm of $|\varphi|_{\tau}$.

In the following result for non-associative vector bundles, the special case for line bundles is implicit in the fundamental work of Gross [17]. Our proof uses an argument of Bakry [4].

Proposition 3.1. Let $a>0, b \geq 0$. The following two conditions are equivalent.

(i) $\left(P_{t}\right)_{t \geq 0}$ possesses hypercontractivity, that is,

$$
\begin{gathered}
\left\|P_{t} \varphi\right\|_{p(t)} \leq e^{m(t)}\|\varphi\|_{p} \quad\left(\varphi \in C_{c}^{\infty}(E)\right) \quad \text { with } \\
p(t)=1+(p-1) e^{\frac{4}{a} t}, \quad m(t)=b\left(p^{-1}-p(t)^{-1}\right) \quad(t>0, p>1) .
\end{gathered}
$$

(ii) For all $p>1$, we have

$$
\operatorname{Ent}\left(|\varphi|_{\tau}^{p}\right) \leq-\left.\frac{a p^{2}}{8(p-1)} \int_{M}|\varphi|_{\tau}^{p-2} \frac{d}{d t}\right|_{t=0}\left|P_{t} \varphi\right|_{\tau}^{2}+b|| \varphi||_{p}^{p} .
$$


Proof. Consider the function $F(t)=e^{-m(t)}\left\|P_{t} \varphi\right\|_{p(t)}$ where $m(0)=0$ and $p(0)=p$. We have $F(0)=\|\varphi\|_{p}$. A straightforward computation shows that

$$
\begin{aligned}
\frac{d}{d t} \log F(t)= & -m^{\prime}(t)+\frac{p^{\prime}(t)}{p(t)^{2}} \frac{1}{\left\|P_{t} \varphi\right\|_{p(t)}^{p(t)}} \operatorname{Ent}\left(\left|P_{t} \varphi\right|_{\tau}^{p(t)}\right) \\
& +\frac{1}{2\left\|P_{t} \varphi\right\|_{p(t)}^{p(t)}} \int_{M}\left|P_{t} \varphi\right|_{\tau}^{p(t)-2} \frac{d}{d t}\left|P_{t} \varphi\right|_{\tau}^{2} .
\end{aligned}
$$

Multiplying both sides by $\left\|P_{t} \varphi\right\|_{p(t)}^{p(t)}$, we obtain

$\quad\left\|P_{t} \varphi\right\|_{p(t)}^{p(t)}\left(\frac{d}{d t} \log F(t)\right)$

$$
=\frac{p^{\prime}(t)}{p^{2}(t)}\left[\operatorname{Ent}\left(\left|P_{t} \varphi\right|_{\tau}^{p(t)}\right)+\frac{p(t)^{2}}{2 p^{\prime}(t)} \int_{M}\left|P_{t} \varphi\right|_{\tau}^{p(t)-2} \frac{d\left|P_{t} \varphi\right|_{\tau}^{2}}{d t}-\frac{m^{\prime}(t) p(t)^{2}}{p^{\prime}(t)}\left\|P_{t} \varphi\right\|_{p(t)}^{p(t)}\right]
$$

By definition, $p(t)$ and $m(t)$ are chosen to solve the following differential equations:

$$
\frac{p(t)^{2}}{p^{\prime}(t)}=\frac{a p^{2}}{4(p-1)}, \quad p(0)=p
$$

and

$$
\frac{m^{\prime}(t) p(t)^{2}}{p^{\prime}(t)}=b, \quad m(0)=0 .
$$

Assume (i). Since $F(0)=\|\varphi\|_{p}$, the hypercontractivity of $\left(P_{t}\right)$ implies $F^{\prime}(0) \leq 0$ which gives, via (8),

$$
\operatorname{Ent}\left(|\varphi|_{\tau}^{p}\right)+\left.\frac{p^{2}}{2 p^{\prime}(0)} \int_{M}|\varphi|_{\tau}^{p-2} \frac{d}{d t}\right|_{t=0}\left|P_{t} \varphi\right|_{\tau}^{2}-\frac{m^{\prime}(0) p^{2}}{p^{\prime}(0)}\|\varphi\|_{p}^{p} \leq 0
$$

Together with (5), this shows (6) holds.

Conversely, assume (ii). Applying (6) to $P_{t} \varphi$ and using (8), we see that (6) implies $\frac{d}{d t} \log F(t) \leq 0$, so $F^{\prime}(t) \leq 0$. Therefore $F(t) \leq F(0)$ which in turn yields the hypercontractivity of $\left(P_{t}\right)_{t \geq 0}$.

Theorem 3.2. Let $\left(P_{t}\right)_{t \geq 0}$ be the diffusion semigroup on a non-associative vector bundle $E \longrightarrow M$ with the generator $L$ associated with the Dirichlet form

$$
\mathcal{E}(\varphi, \psi)=\int_{M}\langle D \varphi, D \psi\rangle_{\tau} d \mu \quad\left(\varphi, \psi \in C_{c}^{\infty}(E)\right) .
$$

Then the hypercontractivity of $\left(P_{t}\right)_{t \geq 0}$ is equivalent to the following log-Sobolev inequality

$$
\operatorname{Ent}\left(|\varphi|_{\tau}^{2}\right) \leq a \int_{M}\langle D \varphi, D \varphi\rangle_{\tau} d \mu+b|| \varphi \|_{2}^{2}
$$


Proof. As

$$
\left.\frac{d}{d t}\right|_{t=0}\left|P_{t} \varphi\right|_{\tau}^{2}(x)=\left.\frac{d}{d t}\right|_{t=0}\left\langle P_{t} \varphi(x), P_{t} \varphi(x)\right\rangle_{x}=2\langle L \varphi(x), \varphi(x)\rangle_{x}
$$

we have

$$
-\left.\int_{M}|\varphi|_{\tau}^{p-2} \frac{d}{d t}\right|_{t=0}\left|P_{t} \varphi\right|_{\tau}^{2} d \mu=2 \int_{M}\left\langle D \varphi, D\left(|\varphi|_{\tau}^{p-2} \varphi\right)\right\rangle_{\tau} d \mu .
$$

For any $\beta>0$, we have by the product rule,

$$
D\left(|\varphi|_{\tau}^{\beta} \varphi\right)=\left(d|\varphi|_{\tau}^{\beta}\right) \varphi+|\varphi|_{\tau}^{\beta} D \varphi
$$

so that

$$
\begin{aligned}
\left|D\left(|\varphi|_{\tau}^{\beta} \varphi\right)\right|_{\tau}^{2} & =\left\langle\left(d|\varphi|_{\tau}^{\beta}\right) \varphi+|\varphi|_{\tau}^{\beta} D \varphi,\left(d|\varphi|_{\tau}^{\beta}\right) \varphi+|\varphi|_{\tau}^{\beta} D \varphi\right\rangle_{\tau} \\
& =\left.\left.|d| \varphi\right|_{\tau} ^{\beta}\right|^{2}|\varphi|_{\tau}^{2}+|\varphi|_{\tau}^{2 \beta}|D \varphi|_{\tau}^{2}+\left\langle D \varphi,\left(d|\varphi|_{\tau}^{2 \beta}\right) \varphi\right\rangle_{\tau}
\end{aligned}
$$

While

$$
\left\langle D \varphi, D\left(|\varphi|_{\tau}^{p-2} \varphi\right)\right\rangle_{\tau}=\left\langle D \varphi,\left(d|\varphi|_{\tau}^{p-2}\right) \varphi\right\rangle_{\tau}+|\varphi|_{\tau}^{p-2}|D \varphi|_{\tau}^{2}
$$

and therefore, with $\beta=(p-2) / 2$, we have

$$
\begin{aligned}
\left\langle D \varphi, D\left(|\varphi|_{\tau}^{p-2} \varphi\right)\right\rangle_{\tau} & =\left|D\left(|\varphi|_{\tau}^{\beta} \varphi\right)\right|_{\tau}^{2}-\left.\left.|d| \varphi\right|_{\tau} ^{\beta}\right|^{2}|\varphi|_{\tau}^{2} \\
& =\left|D\left(|\varphi|_{\tau}^{\frac{p}{2}-1} \varphi\right)\right|_{\tau}^{2}-\left.\left.\frac{(p-2)^{2}}{p^{2}}|d| \varphi\right|_{\tau} ^{\frac{p}{2}}\right|^{2} .
\end{aligned}
$$

Hence, by Proposition 3.1, the hypercontractivity of $\left(P_{t}\right)_{t \geq 0}$ is equivalent to the following entropy inequality:

$$
\operatorname{Ent}\left(|\varphi|_{\tau}^{2}\right) \leq \frac{a p^{2}}{4(p-1)} \int_{M}\left(|D \varphi|_{\tau}^{2}-\left.\left.\frac{(p-2)^{2}}{p^{2}}|d| \varphi\right|_{\tau}\right|^{2}\right)+b|| \varphi \|_{2}^{2}
$$

for all $p>1$ and $\varphi \in C_{c}^{\infty}(E)$. Our claim will follow if we can show for any given $\varphi$, the right-hand side is minimized when $p=2$. To this end we consider

$$
\begin{aligned}
U(p) & =\frac{p^{2}}{p-1} \int_{M}\left(|D \varphi|_{\tau}^{2}-\left.\left.\frac{(p-2)^{2}}{p^{2}}|d| \varphi\right|_{\tau}\right|^{2}\right) \\
& =\frac{p^{2}}{p-1} \int_{M}|D \varphi|_{\tau}^{2}-\left.\left.\frac{(p-2)^{2}}{p-1} \int_{M}|d| \varphi\right|_{\tau}\right|^{2}
\end{aligned}
$$

where it is clear that

$$
U^{\prime}(p)=\frac{p(p-2)}{(p-1)^{2}}\left(\int_{M}|D \varphi|_{\tau}^{2}-\left.\left.\int_{M}|d| \varphi\right|_{\tau}\right|^{2}\right) .
$$

Therefore $U(p)$ takes its minimum value at $p=2$, or at $\int_{M}|D \varphi|_{\tau}^{2}=\left.\left.\int_{M}|d| \varphi\right|_{\tau}\right|^{2}$, where in the latter case, $U(p)$ is constant. In both cases, the minimum value of $U(p)$ is $4 \int_{M}|D \varphi|_{\tau}^{2}$ which proves our claim. 
In the scalar case, the reduction in (6) from any value $p$ to $p=2$ (logarithmic Sobolev inequality) is achieved by the simple fact that $\int_{M}|D \varphi|^{2}=\int_{M}|d| \varphi||^{2}$. The latter is no longer true for sections of vector bundles. Our only contribution is the observation that, nevertheless, such a reduction can still be obtained via a max-min argument instead.

Corollary 3.3. Let $\mu$ be a $\sigma$-finite measure on a Riemannian manifold $M$. If a logarithmic Sobolev inequality holds for functions:

$$
\operatorname{Ent}\left(f^{2}\right) \leq a \int_{M}|\nabla f|^{2}+b|| f \|_{2}^{2} \quad \text { for all } f \in C_{c}^{\infty}(M)
$$

then the semigroup $\left(P_{t}\right)_{t \geq 0}$ on a non-associative vector bundle $E \longrightarrow M$ as in Theorem 3.2 possesses hypercontractivity.

Proof. Since $D$ is compatible with the Riemannian structure on $E$, we have

$$
d|\varphi|^{2}=2\langle D \varphi, \varphi\rangle
$$

so that $\left.\left.|d| \varphi\right|_{\tau} ^{2}|\leq 2| D \varphi\right|_{\tau}|\varphi|_{\tau}$ which implies that $\left.\left.|d| \varphi\right|_{\tau}|\leq| D \varphi\right|_{\tau}$. However $\left.|d| \varphi\right|_{\tau} \mid=$ $\left.|\nabla| \varphi\right|_{\tau} \mid$, therefore by applying (11) to $|\varphi|_{\tau}$, we obtain

$$
\begin{aligned}
\operatorname{Ent}\left(|\varphi|_{\tau}^{2}\right) & \leq\left.\left. a \int_{M}|d| \varphi\right|_{\tau}\right|^{2}+\left.b|| \varphi\right|_{2} ^{2} \\
& \leq a \int_{M}|D \varphi|_{\tau}^{2}+b\|\varphi\|_{2}^{2} .
\end{aligned}
$$

The conclusion now follows from the above theorem immediately.

\section{HARMONIC FUNCTIONS}

To conclude, we discuss harmonic functions with respect to a Dirichlet Laplacian in the scalar case on Lie groups. We show, not surprisingly, the absence of a nontrivial $L^{p}$ harmonic function for $1 \leq p<\infty$.

Let $G$ be a connected Lie group with a right invariant Haar measure $\lambda$, and let $L^{p}(G)$ be the Lebesgue spaces with respect to the Haar measure $\lambda$. Given a Dirichlet form $\mathcal{E}$ on $L^{2}(G)$, we consider the associated positive self-adjoint operator $L$ in $L^{2}(G)$, the Dirichlet Laplacian of $\mathcal{E}$, satisfying

$$
\mathcal{E}(\varphi, \psi)=\langle L \varphi, \psi\rangle \quad(\varphi, \psi \in \mathcal{D}(L)) .
$$

We assume that $L$ commutes with right translations of $G$ :

$$
L r_{a}=r_{a} L \quad(a \in G)
$$

where $r_{a}: x \mapsto x a \in G$ is a right translation by $a$. In this case, the Markov semigroup

$$
P_{t}: L^{p}(G) \longrightarrow L^{p}(G) \quad(t \geq 0)
$$


generated by $L$, commutes with right translations of $G$ and is a convolution semigroup:

$$
P_{t}(f)=f * \sigma_{t} \quad\left(f \in L^{p}(G)\right)
$$

where $\left(\sigma_{t}\right)_{t \geq 0}$ is a family of probability measures on $G$ and the support of each $\sigma_{t}$ generates the group $G$. A complex function $f \in \mathcal{D}(L)$ is called L-harmonic if $L f=0$.

Theorem 4.1. Let $1 \leq p<\infty$ and let $f \in L^{p}(G)$. If $f$ is L-harmonic, then $f$ is constant.

Proof. Let $\left(\sigma_{t}\right)_{t \geq 0}$ be the induced convolution semigroup of probability measures on $G$. Then we have $f * \sigma_{t}=f$ and since the support of $\sigma_{t}$ generates $G$, by $[5$, Theorem 3.12], $f$ is constant.

We note that, given a complete Riemannian manifold $M$ and the Laplace operator $\Delta$ of its Riemannian metric, it is a well-known result of Yau [28] that all $L^{p}$ $\Delta$-harmonic functions on $M$ are constant, for $1<p<\infty$, and if in addition, $M$ has non-negative Ricci curvature, then all $L^{1}$ harmonic functions on $M$ are also constant $[29,22]$ (see also [16]). Yau's result applies to Lie groups for $1<p<\infty$, however, it has been shown by Milnor [23] that for almost all left-invariant Riemannian metrics on a Lie group, the Ricci curvature changes sign and in this case, the above $L^{1}$ result does not apply directly although Theorem 4.1 shows that it is still true for all Lie groups.

\section{Acknowledgment}

The first-named author gratefully acknowledges support of the European Commission through its 6th Framework Programme "Structuring the European Research Area" and the contract RITA-CT-2004-505493, during his visit at IHÉS, France.

\section{REFERENCES}

[1] S. Albeverio and R. H申egh-Krohn, Dirichlet forms and Markov semigroups on $C^{*}$-algebras, Comm. Math. Phys. 56 (1977) 173-187.

[2] Sh. A. Ayupov and R.Z. Abdullaev, On isometries of nonassociative $L_{p}$-spaces, in "Quantum probability and applications IV (Rome 1987)", (Lecture Notes in Math. 1396, SpringerVerlag, Berlin 1989) 99-106.

[3] D. Bakry, L'hypercontractivité et son utilisation en théorie des semigroupes, in "Ecole d'Eté de Probabilités de Saint Flour 1992", Lecture Notes in Math. 1581, Springer-Verlag, Berlin, (1994) 1-114.

[4] D. Bakry, On Sobolev and logarithmic Sobolev inequalities for Markov semigroups, in "New Trends in Stochastic Analysis" (ed. K. D. Elworthy) World Scientific (1997) 43-75.

[5] C-H. Chu, Harmonic function spaces on groups, J. London Math. Soc. 70 (2004) 182-198.

[6] E.B. Davies, Heat kernels and spectral theory, Cambridge Univ. Press, 1989, Cambridge.

[7] E.B. Davies, L. Gross and B. Simon, Hpercontractivity: a bibliogrphic review, in "Ideas and methods in quantum and statistical physics", Cambridge Univ. Press (1992) 370-389.

[8] E.B. Davies and J.M. Lindsay, Non-commutative symmetric Markov semigroups, Math. Z. 210 (1992) 379-411. 
[9] E.B. Davies and O.S. Rothaus, Markov semigroups on $C^{*}$ bundles, J. Funct. Analysis 85 (1989) 264-286.

[10] E.B. Davies and O.S. Rothaus, $A$ BLW inequality for vector bundles and applications to spectral bounds, J. Funct. Analysis 86 (1989) 390-410.

[11] E.B. Davies and B. Simon, Ultracontractivity and the heat kernel for Schödinger operators and Dirichlet Laplacians, J. Func. Analysis 59 (1992) 335-395.

[12] J.-D. Deuschel and D.W. Stroock, Large Deviations, Academic Press, New York, 1989.

[13] J. Diestel and J.J. Uhl, Vector measures, Mathematical Survey 15, Amer. Math. Soc. 1977.

[14] J. Glimm, Boson fields with nonlinear self-interaction in two dimensions, Comm. Math. Phys. 8 (1968) 12-25.

[15] K.R. Goodearl, Notes on real and complex C*-algebras, Shiva, England 1982.

[16] R.E. Greene and $\mathrm{H}$. Wu, Integrals of subharmonic functions on manifolds of nonnegative curvature, Invent. Math. 27 (1974) 265-298.

[17] L. Gross, Logarithmic Sobolev inequalities, Amer. J. Math. 97 (1975) 1061-1083.

[18] L. Gross, Logarithmic Sobolev inequalities and contractivity properties of semigroups, in "Dirichlet forms (Varenna 1992)", Lecture Notes in Math. 1563, Springer-Verlag, Berlin (1993) 54-88.

[19] H.Hanche-Olsen and E. Stormer, Jordan operator algebras, Pitman, London, 1984.

[20] B. Iochum, Non-associative $L^{p}$-spaces, Pacific J. Math. 122 (1986) 417-433.

[21] W. Klingenberg, Riemannian geometry, de Gruyter, Berlin (1982).

[22] P. Li and R. Schoen, $L^{p}$ and mean value properties of subharmonic functions on Riemannian manifolds, Acta Math. 153 (1984) 279-301.

[23] J. Milnor, Curvatures of left invariant metrics on Lie groups, Adv. Math. 21 (1976) 293329.

[24] E. Nelson, A quartic interaction in two dimensions, in "Mathematical Theory of Elementary Particles", 69-73, M.I.T. Press, Cambridge, Mass. 1966.

[25] E. Nelson, The free Markoff field, J. Funct. Analysis, 12 (1973) 211-227.

[26] I.E. Segal, Construction of non-linear local quantum processes I, Ann. of Math. 92 (1970) 462-481.

[27] M. Takesaki, Theory of operator algebras I, Springer-Verlag, Heildelberg, 1979.

[28] S-T. Yau, Some function-theoretic properties of complete Riemannian manifolds and their applications to geometry, Indiana Univ. Math. J. 25 (1976) 659-670.

[29] S-T. Yau, Harmonic functions on complete Riemannian manifolds, Comm. Pure Appl. Math. 28 (1975) 201-228.

IHÉS, F-91440 BurEs-SUR-YVetTe, France

E-mail address: chu@ihes.fr

and

School of Mathematical Sciences, Queen Mary, University of London, LONDON E1 4NS, UK

E-mail address: c.chu@qmul.ac.uk

Mathematical Institute, University of Oxford, Oxford OX1 3LB, UK

E-mail address: qianz@maths.ox.ac.uk 